\title{
Effect of variation in colour gradient information for optic flow computations
}

\author{
B.N. Shobha ${ }^{1}{ }^{*}$, Govind R. Kadambi ${ }^{1}$, S.R. Shankapal ${ }^{1}$, Yuri Vershinim ${ }^{2}$ \\ ${ }^{1}$ MSRSAS, Bangalore \\ ${ }^{2}$ Coventry University, U.K \\ *Corresponding author E-mail: b_n_shobha67@yahoo.com
}

Copyright $\odot 2014$ B.N. Shobha et al. This is an open access article distributed under the Creative Commons Attribution License, which permits unrestricted use, distribution, and reproduction in any medium, provided the original work is properly cited.

\begin{abstract}
Optic flow algorithms provide mapping of 3D velocities on 2D image space. Optic flow is computed on the pair of images which are in sequence and is normally gray scale images. Optic flow computation using Horn and Schunck assumes that brightness consistency is maintained. Colour optic flow has the advantage that optic flow vectors are obtained even when there is a variation of brightness in the input images. The use of colour bands for optic flow is investigated by considering gradients of colour bands and component gradients. Results of applying these two types of gradients to three colour models are presented and analyzed. Decision logic is proposed to select the best colour model for colour optic flow computation based on gradient analysis.
\end{abstract}

Keywords: Activity Measure. Colour Bands, Component Gradients, Decision Logic, Optic Flow Computation.

\section{Introduction}

Optic flow gives important information about the spatial arrangement of the objects viewed and the rate of change of this arrangement. The advancements in the field of computer vision require the analysis of motion estimation. One of the methods of motion estimation requires the computation of optic flow. The computation of optic flow needs to be accurate. The accuracy of the optic flow obtained depends on the noise content in the input image sequences. Accuracy is obtained by removing noise using various filters as a preprocessing stage [1]. The brightness constant constraint of optic flow computation can be overcome by considering only the colour information in the images.

There are different types of optic flow calculation methods like gradient based [2], [3], region based, phase and energy based [4]. Gradient based optical flow vector computation uses spatial and temporal partial derivatives at every position in the image. These gradient based algorithms assume that the apparent velocity of the brightness pattern varies smoothly almost everywhere in the image. Frequency based methods make use of phase and energy information between the consecutive images obtained from a sequence. These techniques are essentially a differential method applied to phase rather than intensity. Although these methods produce accurate results, they are computationally expensive and they are generally not suitable for real-time applications.

\section{Colour information}

Colour models are different ways in which colour information is represented. Each industry requires usage of different colour models of which the most common colour model is RGB. The colour of each pixel is determined by the combination of the red, green and blue intensities stored in each colour plane at the pixel's location. 


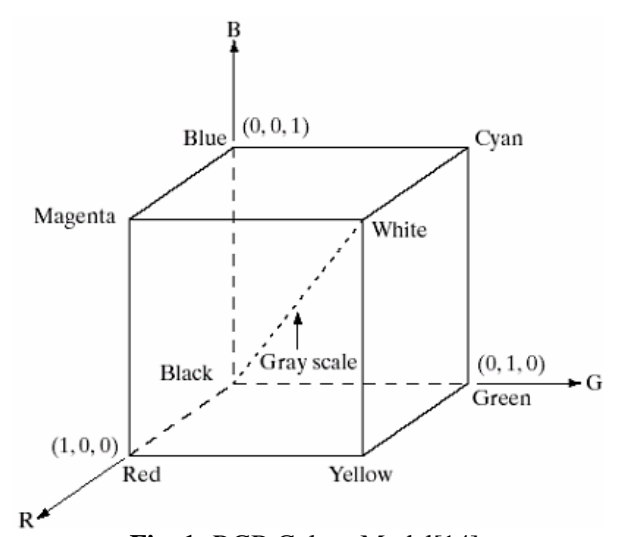

Fig. 1: RGB Colour Model[14]

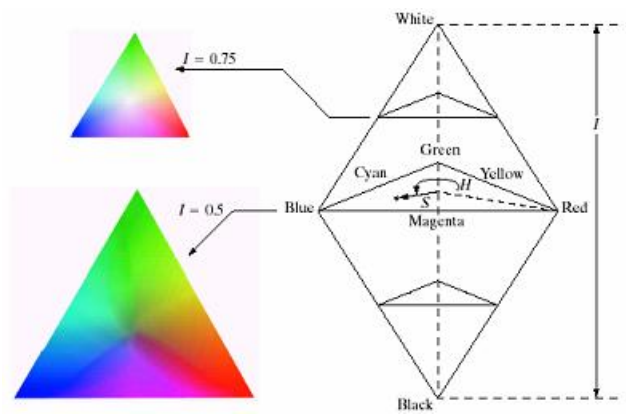

Fig. 2: HIS Colour Model W.R.T RGB[14]

YIQ, YCbCr and HSI are the colour models considered for the analysis purpose. RGB colour model and HIS are shown in fig 1 and fig 2 respectively.

\section{Colour components analysis}

There is less information in literature pertaining to optic flow from colour image gradients. Ohta has investigated the effectiveness of using colour information for optical flow computation by measuring the angle between intensity gradients in different colour bands [5]. Golland has proposed the concept of "colour conservation". By constructing a linear system to solve from only colour components, e.g. Hue and Saturation from the HSV colour model, the illumination in an input sequence for optic flow computation can be varied. The assumption is now the colour conservation rather than brightness conservation [6]. Basic colour image model consist of RGB bands. If gradient eqn is applied to each band the set of eqns (1) are obtained

$$
\begin{aligned}
& R_{x} u+R_{y} v+R_{t}=0 \\
& G_{x} u+G_{y} v+G_{t}=0 \\
& B_{x} u+B_{y} v+B_{t}=0
\end{aligned}
$$

Where

$\mathrm{R}_{\mathrm{x}}, \mathrm{R}_{\mathrm{y}}, \mathrm{R}_{\mathrm{t}}$ are $\mathrm{R}$ band partial derivatives of image coordinates $\mathrm{x}, \mathrm{y}$ and time.

$\mathrm{G}_{\mathrm{x}}, \mathrm{G}_{\mathrm{y}}, \mathrm{G}_{\mathrm{t}}$ are $\mathrm{G}$ band partial derivatives of image coordinates $\mathrm{x}, \mathrm{y}$ and time

$\mathrm{B}_{\mathrm{x}}, \mathrm{B}_{\mathrm{y}}, \mathrm{B}_{\mathrm{t}}$ are $\mathrm{B}$ band partial derivatives of image coordinates $\mathrm{x}, \mathrm{y}$ and time

If any of these two are independent, the flow vector $(\mathrm{u}, \mathrm{v})$ is determined for those two bands. When considering the information about colour, the assumption of flow distribution in space is not required [5]. HSV (Hue, Saturation and Value) model expresses the intensity of the image (V) which is independent of the colour components, Hue and Saturation. Optical flow based purely on V is relying on brightness conservation and methods which are based on Hue and Saturation rely purely on colour conservation. Methods which use intensity and colour information incorporate both assumptions. Three colour models RGB, HSV and normalized RGB have been implemented and tested in [6]. However they have mentioned that YUV and CIE can also be experimented with. The performance of the optical flow techniques vary when images were represented with different colour models. There is a need to find a method for automatically identifying the most appropriate colour model to use for a colour image sequence. The answer to this question may benefit computer vision applications that work on colour image sequences for optic flow computations.

The various colour models that are available are RGB, HSV, YUV, etc. If the intensity components are excluded then the method becomes robust against change in illumination.

Ohta [5] has adopted a direct method in which the angle between the gradients, $\theta$ is taken as the measure as in eqn 2.

$\theta=\angle\left(\nabla \mathrm{I}_{1}, \nabla \mathrm{I}_{2}\right)$

Where

$I_{1}$ and $I_{2}$ in eqn 2 are taken from $R, G$ and $B$, respectively.

If angle $\theta$ is wider, the two gradients are regarded as more independent. If the length of either gradient is small, the gradient information is not effective because of the presence of image noise. Ohta computed the spatial gradient $\mathrm{VI}$ at position $(\mathrm{x}, \mathrm{y})$ using the formula as shown in eqn 3.

$\nabla \mathrm{I}(\mathrm{x}, \mathrm{y})=\left(\begin{array}{l}\mathrm{I}(\mathrm{x}+1, \mathrm{y})-\mathrm{I}(\mathrm{x}, \mathrm{y}) \\ \mathrm{I}(\mathrm{x}, \mathrm{y}+1)-\mathrm{I}(\mathrm{x}, \mathrm{y})\end{array}\right)$

Ohta compute angle $\theta$ when both of the gradient lengths are larger than threshold $\mathrm{T}$ as shown in eqn 4 .

$\left\|\nabla \mathrm{I}_{1}\right\|>\mathrm{T}$ and $\left\|\nabla \mathrm{I}_{2}\right\|>\mathrm{T}$ 
Ohta has investigated angle $\theta$ for R, G and B bands. He has suggested the result is valid for other colour representations such as HSV. They have investigated the effectiveness of using colour information for optical flow computation by measuring the angle between intensity gradients in different colour bands of RGB. Their results show that for some colourful objects like flowers, using colour information is effective, but for many other objects, it is not necessarily so.

The same investigation has been made for multi-spectral images [7], which contain more precise spectral information; there is a possibility that the results were different. Moreover, in the case that infrared images are used as one band images, it is expected that more different gradients can be obtained, because those images reflect a different physical phenomenon i.e. temperature of objects. In this paper the gradient concept is considered and applied it to the HIS, YIQ and $\mathrm{YCbCr}$.

Consider two colour components $\mathrm{U}$ and $\mathrm{V}$ that are converted from $\mathrm{R}, \mathrm{G}$ and $\mathrm{B}$ by functions $\mathrm{f}$ and $\mathrm{g}$ respectively as shown in eqn 5 . The space gradients of these components are given in eqn 6 :

$\mathrm{U}=\mathrm{f}(\mathrm{R}, \mathrm{G}, \mathrm{B})$

$\mathrm{V}=\mathrm{g}(\mathrm{R}, \mathrm{G}, \mathrm{B})$

$\nabla \mathrm{U}=\frac{\partial \mathrm{f}}{\partial \mathrm{R}} \nabla \mathrm{R}+\frac{\partial \mathrm{f}}{\partial \mathrm{G}} \nabla \mathrm{G}+\frac{\partial \mathrm{f}}{\partial \mathrm{B}} \nabla \mathrm{B}$

$\left.\nabla \mathrm{V}=\frac{\partial \mathrm{g}}{\partial \mathrm{R}} \nabla \mathrm{R}+\frac{\partial \mathrm{g}}{\partial \mathrm{G}} \nabla \mathrm{G}+\frac{\partial \mathrm{g}}{\partial \mathrm{B}} \nabla \mathrm{B}\right)$

The eqns shows that $\nabla \mathrm{U}$ and $\nabla \mathrm{V}$ are represented by linear combinations of $\nabla \mathrm{R}, \nabla \mathrm{G}$ and $\nabla \mathrm{B}$ if $\nabla \mathrm{R}, \nabla \mathrm{G}$ and $\nabla \mathrm{B}$ are not independent $\nabla \mathrm{U}$ and $\nabla \mathrm{V}$ cannot be independent [5]. The component analysis is carried out as shown in eqns 7 .

$\nabla \mathrm{U}=\mathrm{i}\left[\frac{\partial \mathrm{f}}{\partial \mathrm{R}} \mathrm{Rx}+\frac{\partial \mathrm{f}}{\partial \mathrm{G}} \mathrm{Gx}+\frac{\partial \mathrm{f}}{\partial \mathrm{B}} \mathrm{Bx}\right]+\mathrm{j}\left[\frac{\partial \mathrm{f}}{\partial \mathrm{R}} \mathrm{Ry}+\frac{\partial \mathrm{f}}{\partial \mathrm{G}} \mathrm{Gy}+\frac{\partial \mathrm{f}}{\partial \mathrm{B}} \mathrm{By}\right]$
$\nabla \mathrm{V}=\mathrm{i}\left[\frac{\partial \mathrm{g}}{\partial \mathrm{R}} \mathrm{R} \mathrm{x}+\frac{\partial \mathrm{g}}{\partial \mathrm{G}} \mathrm{Gx}+\frac{\partial \mathrm{g}}{\partial \mathrm{B}} \mathrm{Bx}\right]+\mathrm{j}\left[\frac{\partial \mathrm{g}}{\partial \mathrm{R}} \mathrm{Ry}+\frac{\partial \mathrm{g}}{\partial \mathrm{G}} \mathrm{Gy}+\frac{\partial \mathrm{g}}{\partial \mathrm{B}} \mathrm{By}\right]$

This can be further represented as in eqns 8

$$
\begin{aligned}
& \mathrm{Ux}=\left[\frac{\partial \mathrm{f}}{\partial \mathrm{R}} \mathrm{Rx}+\frac{\partial \mathrm{f}}{\partial \mathrm{G}} \mathrm{Gx}+\frac{\partial \mathrm{f}}{\partial \mathrm{B}} \mathrm{Bx}\right] \\
& \mathrm{Uy}=\left[\frac{\partial \mathrm{f}}{\partial \mathrm{R}} \mathrm{Ry}+\frac{\partial \mathrm{f}}{\partial \mathrm{G}} \mathrm{Gy}+\frac{\partial \mathrm{f}}{\partial \mathrm{B}} \mathrm{By}\right] \\
& \mathrm{Vx}=\left[\frac{\partial \mathrm{g}}{\partial \mathrm{R}} \mathrm{Rx}+\frac{\partial \mathrm{g}}{\partial \mathrm{G}} \mathrm{Gx}+\frac{\partial \mathrm{g}}{\partial \mathrm{B}} \mathrm{Bx}\right] \\
& \mathrm{Vy}=\left[\frac{\partial \mathrm{g}}{\partial \mathrm{R}} \mathrm{Ry}+\frac{\partial \mathrm{g}}{\partial \mathrm{G}} \mathrm{Gy}+\frac{\partial \mathrm{g}}{\partial \mathrm{B}} \mathrm{By}\right] \\
& \theta^{\mathrm{U}}=\tan ^{-1}\left[\frac{\left|\mathrm{U}_{\mathrm{y}}\right|}{\left|\mathrm{U}_{\mathrm{x}}\right|}\right] \\
& \theta^{\mathrm{V}}=\tan ^{-1}\left[\frac{\left|\mathrm{V}_{\mathrm{y}}\right|}{\left|\mathrm{V}_{\mathrm{x}}\right|}\right] \\
& \theta^{\mathrm{U}-\mathrm{V}}=\mid \theta^{\mathrm{U}}-\theta^{\mathrm{V} \mid}
\end{aligned}
$$

$\mathrm{V} \rightarrow \mathrm{Q} \rightarrow \mathrm{g} 1=0.211 \times \mathrm{R}-0.523 \times \mathrm{G}+0,312 \times \mathrm{B}$

$\mathrm{Y}$ is Intensity, $\mathrm{Cb}$ and $\mathrm{Cr}$ carry colour information. In terms of $\mathrm{U}$ and $\mathrm{V}$, they are represented as in eqns 13.

$\mathrm{U} \rightarrow \mathrm{Cb} \rightarrow \mathrm{f} 2=128+(-37.797 \times \mathrm{R})+(-74.203 \times \mathrm{G})+(112 \times \mathrm{B})$

$\mathrm{V} \rightarrow \mathrm{Cr} \rightarrow \mathrm{g} 2=128+(112 \times \mathrm{R})+(-93.786 \times \mathrm{G})+(18.214 \times \mathrm{B})$

$\mathrm{I}$ is Intensity, $\mathrm{H}$ and $\mathrm{S}$ carry colour information, In terms of $\mathrm{U}$ and $\mathrm{V}$, they are represented as in eqns 14. 


$$
\begin{aligned}
& \mathrm{U} \rightarrow \mathrm{H} \rightarrow \mathrm{f} 3=\theta \quad \text { if } \quad \mathrm{B} \leq \mathrm{G} \quad \text { else } 360-\theta \\
& \mathrm{V} \rightarrow \mathrm{S} \rightarrow \mathrm{g} 3=1-\frac{3}{(\mathrm{R}+\mathrm{G}+\mathrm{B})}[\min (\mathrm{RGB})] \\
& \text { where } \theta=\cos ^{-1}\left\{\frac{\frac{1}{2}[(\mathrm{R}-\mathrm{G})+(\mathrm{R}-\mathrm{B})]}{\sqrt{\left[(\mathrm{R}-\mathrm{G})^{2}+(\mathrm{R}-\mathrm{B})() \mathrm{G}-\mathrm{B}\right]}}\right\}
\end{aligned}
$$

\section{Experimentation}

Three sets of colour images are taken as test images corresponding to flowers, scenery and indoor as shown in fig 3 , fig 4 and fig 5.

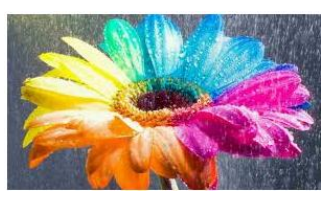

Flower1: $168 \times 300$

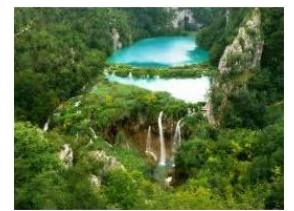

Scenery1:194x259

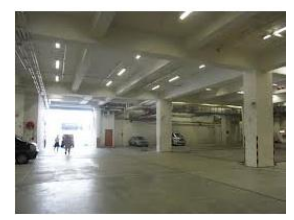

Indoor1: 194x259

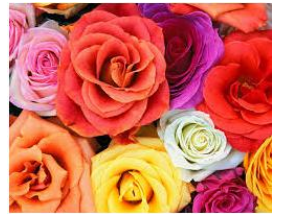

Flower2: 201x251

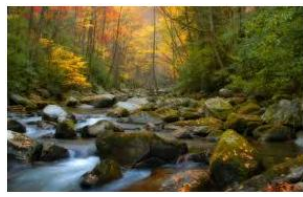

Scenery2:177x284

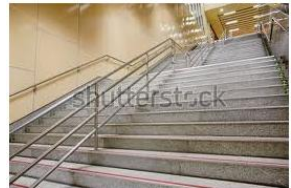

Indoor2: 189x267

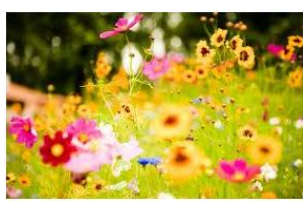

Flower3: $177 \times 284$

Fig. 3: Set of Flower Images

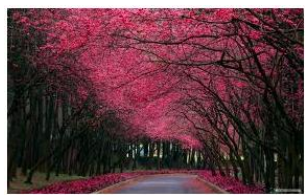

Scenery3: $177 \times 284$

Fig. 4: Set f Scenery Images

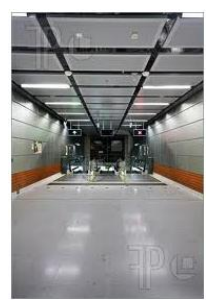

Indoor3: $275 \times 183$

Fig. 5: Set of Indoor Images

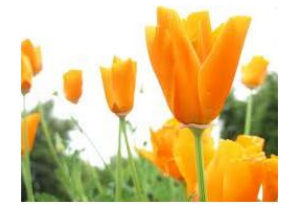

Flower4: $205 \times 246$

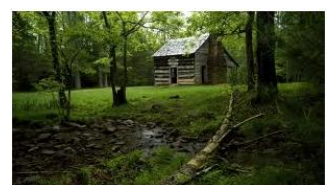

Scenery4: 168x300

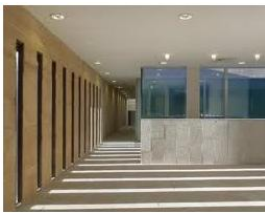

Indoor4: 198x254

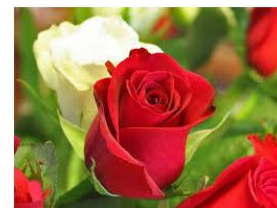

Flower5: 194x259

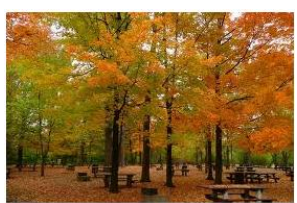

Scenery5: $183 \times 275$

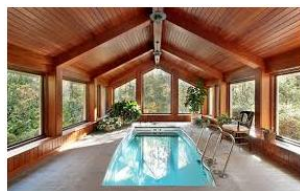

Indoor5: 177x284

\section{Simulation results}

Fig 6 is the test image considered for the experimentation purpose. Fig 7 describes the graphs for theta along the $\mathrm{X}$ axis obtained by using eqn 9 .

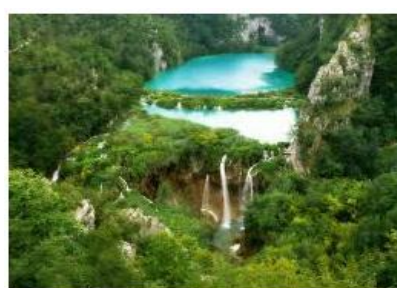

Fig. 6: Sample Image

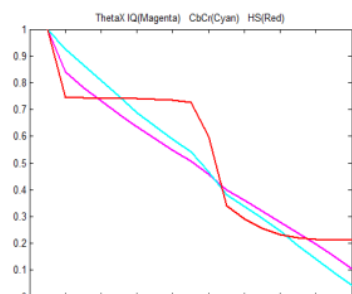

Fig. 7: $\Theta$ Along X Dir

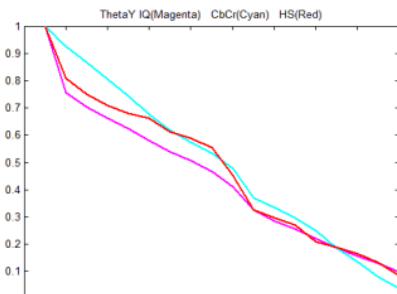

Fig. 8: $\Theta$ Along Y Dir

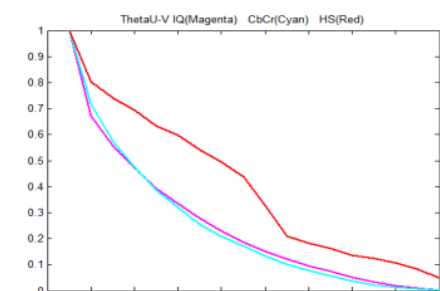

Fig. 9: Diff In $\Theta$ Between X\&Y Dir

Fig 8 describes the graphs for theta along the $\mathrm{Y}$ axis obtained by using eqn 10. Fig 9 describes the graphs for difference in theta between $\mathrm{X} \& \mathrm{Y}$ directions obtained by using eqn 11. In the fig 7, fig 8 and fig 9, graph representing YIQ color model is represented by magenta color, graph representing $\mathrm{YCbCr}$ color model is represented by cyan color and graph representing HSI color model is represented by red color. $\mathrm{X}$ axis represents the angle theta in degrees which varies from 0 to 90 and $\mathrm{Y}$ axis represents the cumulative percentage of image pixels at which the angle $\theta$ is greater than the angle 
indicated by the horizontal axis. For example, if a line indicates 4.0 in the vertical axis at 20 in the horizontal axis, it means that 40 percent of the whole image area has values of $\theta$ greater than 20 degrees [5].

\section{Decision logic}

To identify which colour model has the highest activity measure which means more variation in the gradients, we have considered the area under various curves as shown in eqn 15.

$$
\begin{aligned}
& \theta_{\mathrm{YIQ}}^{\mathrm{U}} \text { curve }=\mathrm{A} \theta_{\mathrm{YIQ}}^{\mathrm{U}} \\
& \theta_{\mathrm{YIQ}}^{\mathrm{V}} \text { curve }=\mathrm{A} \theta_{\mathrm{YIQ}}^{\mathrm{V}} \\
& \theta_{\mathrm{YIQ}}^{\mathrm{U}-\mathrm{V}} \text { curve }=\mathrm{A} \theta_{\mathrm{YIQ}}^{\mathrm{U}-\mathrm{V}} \\
& \theta_{\mathrm{YCbCr}}^{\mathrm{U}} \text { curve }=\mathrm{A} \theta_{\mathrm{YCbCr}}^{\mathrm{U}} \\
& \theta_{\mathrm{YCbCr}}^{\mathrm{V}} \text { curve }=\mathrm{A} \theta_{\mathrm{YCbCr}}^{\mathrm{V}} \\
& \theta_{\mathrm{YCbCr}}^{\mathrm{U}-\mathrm{V}} \text { curve }=\mathrm{A} \theta_{\mathrm{YCbCr}}^{\mathrm{U}-\mathrm{V}} \\
& \theta_{\mathrm{HSI}}^{\mathrm{U}} \text { curve }=\mathrm{A} \theta_{\mathrm{HSI}}^{\mathrm{U}} \\
& \theta_{\mathrm{HSI}}^{\mathrm{V}} \text { curve }=\mathrm{A} \theta_{\mathrm{HSI}}^{\mathrm{V}} \\
& \theta_{\mathrm{HSI}}^{\mathrm{U}-\mathrm{V}} \text { curve }=\mathrm{A} \theta_{\mathrm{HSI}}^{\mathrm{U}-\mathrm{V}}
\end{aligned}
$$

Areas under three curves corresponding to $\theta^{\mathrm{u}}, \theta^{\mathrm{v}}$ and $\theta^{\mathrm{u}-\mathrm{v}}$ for each model are added as shown in eqn 16 and the model which has the maximum area is considered the optimal model for the given input colour image.

$$
\begin{aligned}
& \mathrm{A}_{\mathrm{YIQ}}=\mathrm{A} \theta_{\mathrm{YIQ}}^{\mathrm{U}}+\mathrm{A} \theta_{\mathrm{YIQ}}^{\mathrm{V}}+\mathrm{A} \theta_{\mathrm{YIQ}}^{\mathrm{U}-\mathrm{V}} \\
& \mathrm{A}_{\mathrm{YCbCr}}=\mathrm{A} \theta_{\mathrm{YCbCr}}^{\mathrm{U}}+\mathrm{A} \theta_{\mathrm{YCbCr}}^{\mathrm{V}}+\mathrm{A} \theta_{\mathrm{YCbCr}}^{\mathrm{U}-\mathrm{V}} \\
& \mathrm{A}_{\mathrm{HSI}}=\mathrm{A} \theta_{\mathrm{HSI}}^{\mathrm{U}}+\mathrm{A} \theta_{\mathrm{HSI}}^{\mathrm{V}}+\mathrm{A} \theta_{\mathrm{HSI}}^{\mathrm{U}-\mathrm{V}} \\
& \mathrm{M}=\max \left(\mathrm{A}_{\mathrm{YIQ}}, \mathrm{A}_{\mathrm{YCbCr}}, \mathrm{A}_{\mathrm{HSI}}\right)
\end{aligned}
$$

$\mathrm{M}$ given in eqn 17 is the maximum of the three areas as given in eqn 16. $\mathrm{M}$ is considered the optimal model for the given input colour image.

\section{Conclusions}

A method to identify the color model for optic flow computation of a colour image has been proposed. The method is based on the gradient components of the pixels of a given colour image. Only three colour models YIQ, YCbCr and HSI have been considered, the concept can be extended to any number of colour models. The model selected depends on the colour variation of the input image considered. The selected colour model needs to be applied to a given image sequence, optic flow computed for computer vision application to completely validate the results. As a future enhancement we are working on the automatic selection of colour for the given input image based on the gradient information of the colour bands.

\section{Acknowledgment}

All test images have been taken from google.com

\section{References}

[1] B. N. Shobha, S. R. Shankapal, Govind R. Kadambi, A Performance Characterization of Advanced Data Smoothing Techniques Used for Smoothing Images in Optic Flow Computations, International Journal of Advanced Computer and Mathematical Sciences, Volume 3, Issue 1, 2012, pp 186-193.

[2] B.K.P. Horn and B. G Schunck, Determining Optical Flow, Artificial Intelligence, Vol. 17, 1981, pp 185-203 http://dx.doi.org/10.1016/00043702(81)90024-2

[3] B. D. Lucas and T. Kanade, An Iterative Registration Technique with an Application to Stereo Vision, Proc. 7th Int. Joint Conf. Artificial Intelligence. Vancouver, 1981, pp.674-679,

[4] S. S Beauchemin and J. L Barron, The Computation of Optical Flow, Dept. of Computer Science, University of Western Ontario London Ontario Canada N6A 5B7, 1995. 
[5] Naoya Ohta and Satoe Nishizawa, How Much Does Colour Information Help Optical Flow Computation?, IEICE Transactions on INF. \& SYST., Vol.E89-D, No.5, May 2006, pp. 1759.

[6] P. Golland and A. M. Bruckstein, Motion from colour, Tech. report, Computer Science Dept, Technion, I.I.T., Haifa, Israel, 1997.

[7] Vishal Markandey, Bruce E. Flinchbaugh, Multispectral constraints for optical flow computation. ICCV 1990: 38-41 Osaka, Japan, December 1990, pp. 38-41. 